\title{
Numerical Uncertainty in Density Estimation for Background Oriented Schlieren
}

\author{
Jiacheng Zhang ${ }^{1 \S}$, Lalit K. Rajendran ${ }^{2 \S}$, Sally P. M. Bane ${ }^{2}$, Pavlos P. Vlachos ${ }^{1^{*}}$ \\ ${ }^{1}$ Purdue University, School of Mechanical Engineering, West Lafayette, USA. \\ ${ }^{2}$ Purdue University, School of Aeronautics and Astronautics, West Lafayette, USA. \\ *pvlachos@purdue.edu
}

\section{Abstract}

Background Oriented Schlieren (BOS) is an image-based density measurement technique. BOS estimates the density gradient from the apparent distortion of a target pattern viewed through a medium with varying density using cross-correlation, tracking, or optical flow algorithms. The density gradient can then be numerically integrated to yield a spatially resolved estimate of the density [1]. A method was recently proposed to estimate the a-posteriori instantaneous and spatially resolved density uncertainty for BOS [2] and showed good agreement between the propagated uncertainties and the random error. However, the density uncertainty quantification method could not account for the systematic uncertainty in the density field due to the discretization errors introduced during the numerical integration, which could be much larger than the displacement random errors [2]. In this work, we propose a method to estimate the numerical uncertainty introduced by the density integration in BOS measurements, using a Richardson extrapolation framework. A procedure is also introduced to combine this systematic uncertainty with the random uncertainty from the previous work to provide an instantaneous, spatially-resolved total uncertainty on the density estimates. The method will be tested with synthetic fields and synthetic BOS images.

With the Richardson extrapolation [3], the discretization error of a numerical estimation can be estimated based on the residual between two sets of results with different grid levels as:

$$
\bar{\epsilon}_{h}=-\frac{f_{h}-f_{r h}}{r^{p}-1}
$$

where $\bar{\epsilon}_{h}$ is the estimated numerical error of the result obtained on a grid with spacing $h, f_{h}$ and $f_{r h}$ are the results obtained on the grids with spacing $h$ and $r h$, respectively, with $r$ being the downsampling factor (usually $r=2$ ), and $p$ is the order of accuracy of the discretization scheme. In this study, $p$ is 2 since the second-order central differencing scheme was used for carrying the numerical integration. The estimated error is then employed as the numerical uncertainty $\left(U=\left|\epsilon_{h}\right|\right)$. With the random uncertainty obtained using the previous method [2] as the standard deviation of the random error distribution, and the numerical uncertainty interpreted as the standard deviation of the bias error distribution, the standard total uncertainty can be expressed as

$$
U_{\text {total }}^{2}=U_{\text {bias }}^{2}+U_{\text {random }}^{2}
$$

thereby providing a framework for combining the random uncertainty estimates to estimate the overall uncertainty in the density integration.

The proposed uncertainty estimation method was tested using a synthetic sinusoidal scalar field as:

$$
f(X, Y)=\sin \left(\frac{2 \pi}{\lambda} X\right) \sin \frac{2 \pi}{\lambda} Y,
$$

where $f$ represents the scalar field, and $\lambda$ represents the wavelength. A zero-mean Gaussian distributed noise was added to the scalar field with prescribed noise levels. One thousand (1000) realizations of the corrupted field were generated, and for each realization, the integration was performed with the noisy gradient fields to estimate the error. The results are shown in figure 1 for two noise levels: $1 \%$ and $10 \%$ of the peak value of the scalar field. It is seen in both levels that the spatial variation of the total uncertainty matches that of the total error, and the RMS of the total uncertainty coincides with the RMS of the error distribution. This validates the framework used to combine the bias and random uncertainty estimates.

\footnotetext{
$\S$ These authors contributed equally to this work.
} 

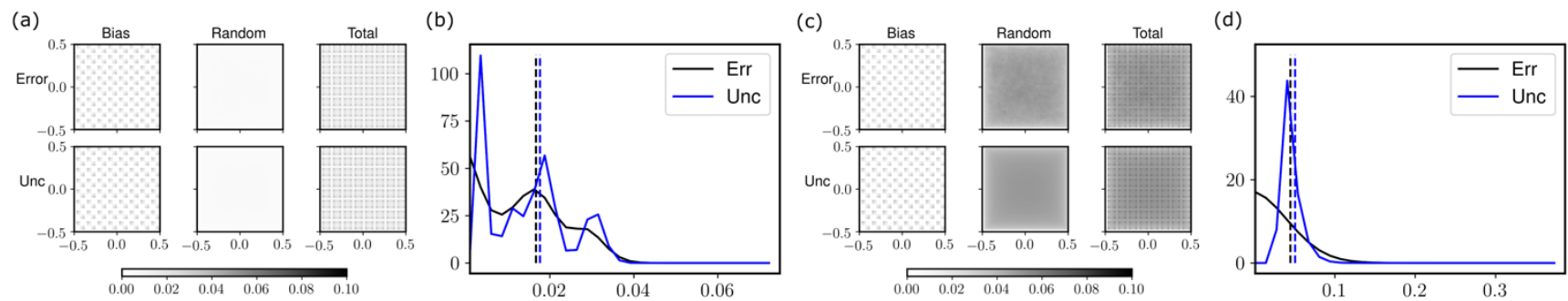

Figure 1 Error and uncertainty statistics for the sinusoidal field for two noise levels. (a) and (b) represent the spatial variation of the statistics and probability density functions respectively for a $1 \%$ noise level, with (c) and (d) representing the results for the $10 \%$ noise level.

The numerical uncertainty estimation was also applied to the synthetic BOS images rendered using a raytracing based image generation methodology [4]. A sinusoidal density field was chosen for the error analysis as described by equation (4):

$$
\rho(X, Y)=\rho_{0}+\Delta \rho_{0} \cos \left(\frac{2 \pi}{\lambda} X\right) \cos \left(\frac{2 \pi}{\lambda} Y\right),
$$

where $\rho_{0}$ is the ambient density, $\Delta \rho_{0}$ is the peak density difference and $\lambda$ is the wavelength. A 2D slice of the density field is shown in figure 2(a). The rendered images were processed using PRANA with a standard crosscorrelation procedure for two passes in an iterative window deformation framework. A sample instantaneous displacement field are shown in figure 2(b). The displacement fields were used to calculate the depth averaged density gradient field $\nabla \rho$, which were then spatially integrated using the Poisson solver to obtain the projected density field. The error of the density field was determined as the deviation from the original density field used to render the synthetic images, and the numerical uncertainty was estimated using the Richardson extrapolation method. The results of the density error and uncertainty are compared in figure 2(c) and (d). The numerical uncertainty of the density integration was lower than the total density error, because the density error was also due to the discrepancy between the ray tracing displacements and the theoretical displacements because of the linear approximation of the non-linear ray trajectory through density gradients [4].

Efforts are ongoing to apply the proposed method to experimental BOS data.
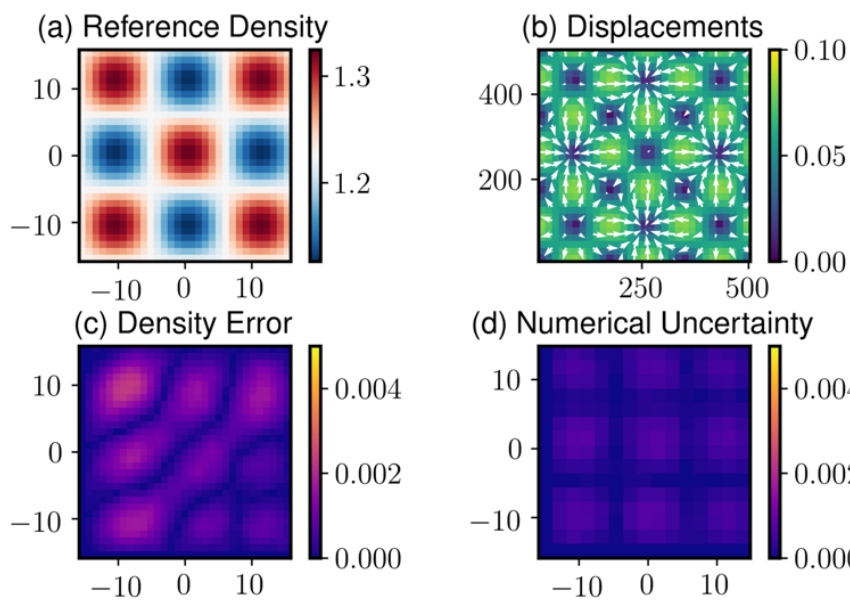

(d) Numerical Uncertainty

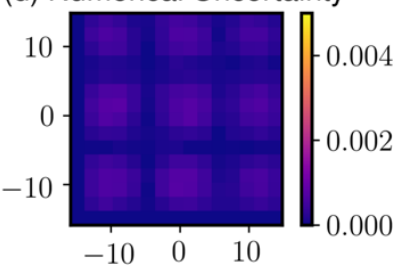

Figure 2 Results of the analysis with synthetic BOS images. (a) 2D slice of the density field used to render the synthetic BOS images, (b) image displacements from cross-correlation analysis, (c) error in the density field, and (d) numerical uncertainty

\section{References}

[1] M. Raffel, "Background-oriented schlieren (BOS) techniques," Exp. Fluids, vol. 56, no. 3, pp. 1-17, 2015.

[2] L. K. Rajendran, J. Zhang, S. Bhattacharya, S. P. M. Bane, and P. P. Vlachos, "Uncertainty quantification in density estimation from background-oriented Schlieren measurements," Meas. Sci. Technol., vol. 31, no. $5,2020$.

[3] P. J. Roache and P. M. Knupp, "Completed Richardson extrapolation," Commun. Numer. Methods Eng., vol. 9, no. 5, pp. 365-374, 1993.

[4] L. K. Rajendran, S. P. M. Bane, and P. P. Vlachos, "PIV/BOS synthetic image generation in variable density environments for error analysis and experiment design," Meas. Sci. Technol., vol. 30, no. 8, 2019. 